\title{
Prototype Development of an App for Choong-Jang-Ro Activation based on Augmented Reality
}

\author{
Seong-Ho Lee ${ }^{1}$, JongMin Moon ${ }^{1}$, Hyeo-Won No ${ }^{1}$, Su-Yong Lee ${ }^{2}$, \\ HyeongGyun $\mathrm{Kim}^{3}$ and Ki-Bong Joung ${ }^{1}$ \\ ${ }^{1}$ Universal Community Co. Ltd. in Korea, ${ }^{2}$ Chonnam National University, \\ ${ }^{3}$ Chosun University \\ wideview06@hanmail.net,whdals0@naver.com,flash200@hanmail.net, \\ leesyarchi@gmail.com,kjdmct@nate.com,topman21@nate.com
}

\begin{abstract}
Choong-Jang-Ro has been a central place for a long time in Gwang-ju, but it was on the decline recently. For activation of Choong-Jang-Ro, an app to let people know it on state-ofthe-art devices needs to be developed. To achieve this objective, we propose the prototype design of an app for Choong-Jang-Ro. Our proposed app has 4 services including a story telling service, a service to provide infor nation relatedto Choong-Jang-Ro, a social network service, and a service to recommend tour course Our app utilizes a technology of augmented reality. We implement our app based on the design of user interface and database. In the future, we will upgrade our app to daunch gn atcommercial market.
\end{abstract}

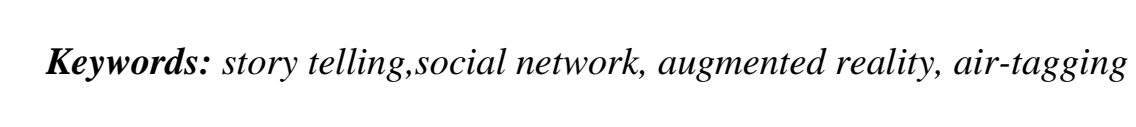

\section{Introduction}

Choong-Jang-Ro is one of the most famous places for tourists to go sightseeing, while they visited Gwang-ju. With Geum Nam-Ro and Mt. Moo-Deung, it is a symbol of Gwang-ju. Until 1990s, it has been the greatest commercial area in Gwang-ju. In recent times, it has been converted to a significant place of culture-centered city by the construction of Asian Culture Complex [1].

As many new residential districts have been formed throughout Gwang-ju since the mid1990s, Choong-Jang-Ro has been on the decline. Because of the development of several commercial areas in Gwang-ju, people over their 30s has not come by Choong-Jang-Ro well. So, itis full of youngsters in their $10 \mathrm{~s}$ and $20 \mathrm{~s}$. That means that it has been transformed into youngster's road.

For the activation of Choong-Jang-Ro, a service to let people know it should be provided. Furthermore, the service should be executed on state-of-the-art devices such as smart phones and tablet PCs because youngsters find information using those devices. Therefore, the service should involve story telling to recall the memory of Choong-Jang-Ro and the service to provide information with respect to culture events and shopping.

To achieve this objective, we propose an app to let people know Choong-Jang-Ro. This paper presents prototype design of the proposed app. This paper is organized as follows. In Section 2, we show the schematic outline of the proposed app. Then, we describe the technology applied to our app in Section 3. Finally, we conclude our paper and describe future work in Section 4. 


\section{Concept design}

\subsection{Schematic outline}

In this section, we show the schematic outline of our app. The objective of our app is to digitalize various memory and information of Choong-Jang-Ro. Our proposed app has 4 services. First, we develop a story telling service for the memory of Choong-Jang-Ro using augmented reality. Augmented Reality (AR) is a live, direct or indirect, view of a physical, real-world environment whose elements are augmented (or supplemented) by computergenerated sensory input such as sound, video, graphics or GPS data [2]. Secondly, we provide information service based on AR. What our app provides is the information related to famous restaurants, public performance, and shopping. Thirdly, we develop a social,network service by using Air tagging. Finally, we provide a service to recommend tøur course. Figure 1 shows the schematic outline of our app.

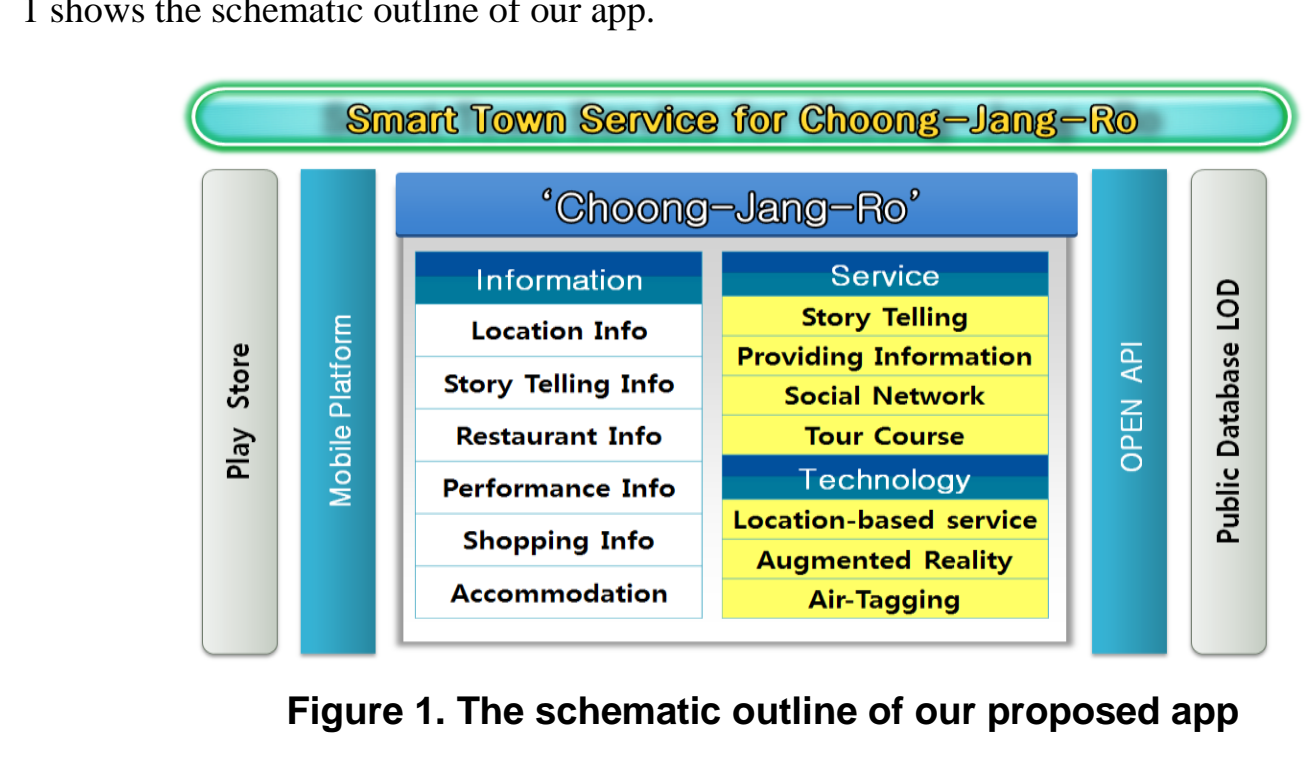

\subsection{Technology applied to our app}

We develop the proposed app using 3 techniques. They are location-based service, augmented reality, and air-tagging. First, we utilize location-based service module to guide a direction to a certain place according to GPS information. If someone clicks a certain place on a LBS tour map, our app will display a right direction from current position to that place on a smart phone. Secondly, augmented reality is used to show proper information pertaining to menoria places. If someone clicks a memorial place on a Choong-Jang-Ro map, we will make oppear its history, picture, and related information. Air-tagging is a kind of augmented reality. If someone takes a picture of a place located on Choong-Jang-Ro after executing our app, several postings which past visitors left are displayed.

We obtain GPS information through GPS sensor, gyro sensor, and camera. Then, this information is delivered to LBS module. LBS module consists of G-Info receive function and G-Info matching function. LBS module shows proper direction on LBS tour map using Open API module. We match currently read data to stored data at GPS DB. Augmented reality module consists of LBS module call function and vision-info receive function. According to the vision received from a camera of user's smart phone, AR module displays data related to the vision. Air-tagging module consists of tag location matching function and air tag 
coordinate function. Air-tagging module searches past-written tags from SNS-Tag DB and shows them on a screen. Figure 2 shows technical components and flow diagram.

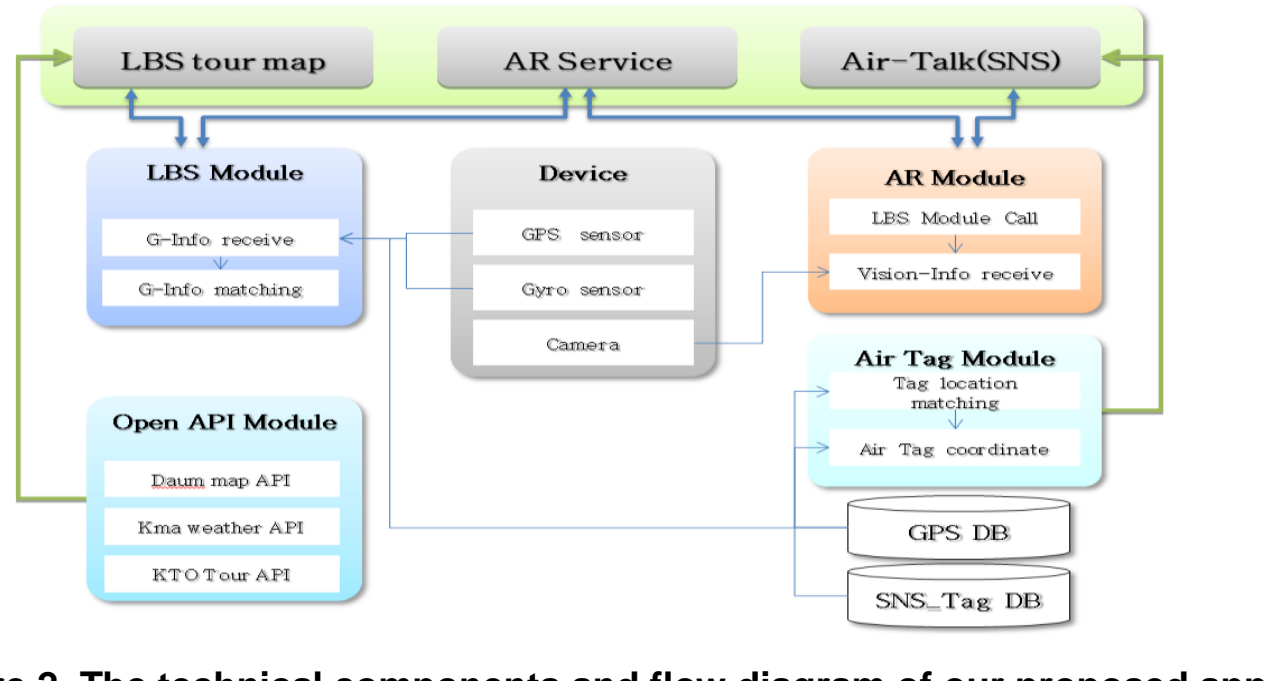

Figure 2. The technical components and flow diagram of our proposed app

\section{The design of user interface}

Figure 3 shows the intro page of but app. If a user press "Start" button, he will enter the start page of our app. Otherwise, if a user.press "Finish" button, our app ends. When a user leave his message within ougapp, he should be logged in. For such a case, it is necessary to register himself as a user inour app.

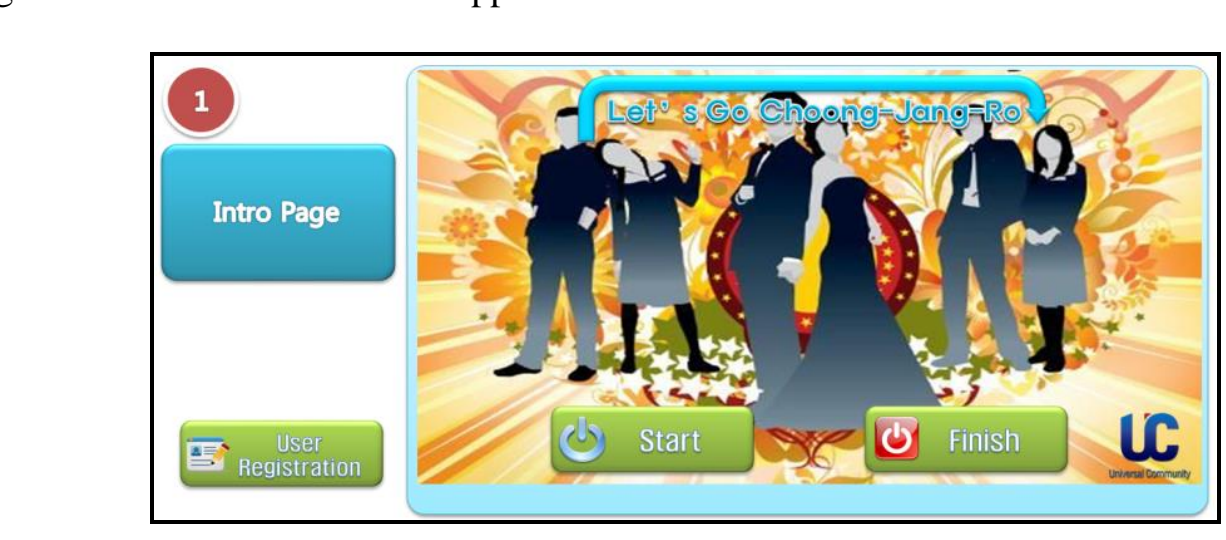

Figure 3. The intro page of our app

Our app will begin the process of registering a user, when a user press "User Registration" button in intro page. Figure 4 shows the user registration page of our app. A user should fill in his information on the blank of a user registration page. Basically, a user should enter name, ID, SSN, password. To confirm the password you enter, a user has to enter password once again. If two passwords are different, a user will see an error page on popup window. To authenticate a user, our app needs the phone number of a user and telecommunication service company which a usre join. 


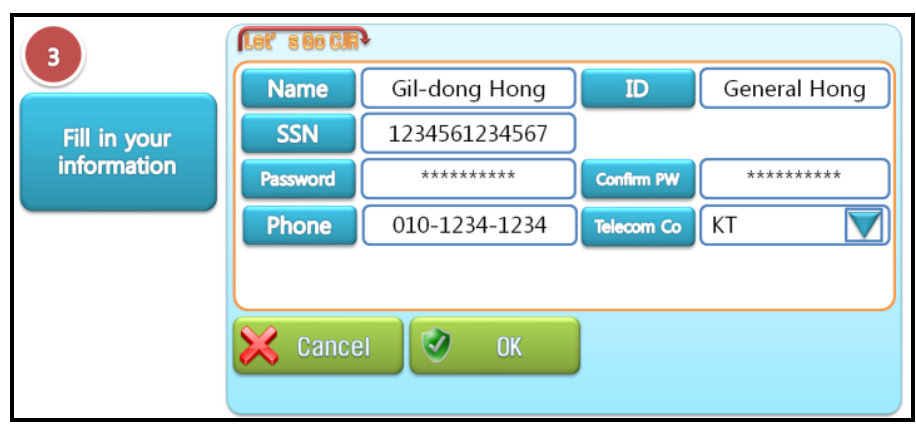

Figure 4. The user registration page of our app

Our app will enter a start page when a user press "Start" button in intro page. Figure 5 shows the start page of our app. If a user press "Log-in" button, our app will begin the process of logging in. In case that a user forget your password and are going to find $\mathrm{it}$, he should press "Find PW" button.

Our app will enter a log-ißpage when a user press "Log-in" button in start page. Figure 6 shows the log-in page of Oyr app. If user's input is correct when he enter user name and password, he will enteratmain page. Otherwise, he will see an error page.

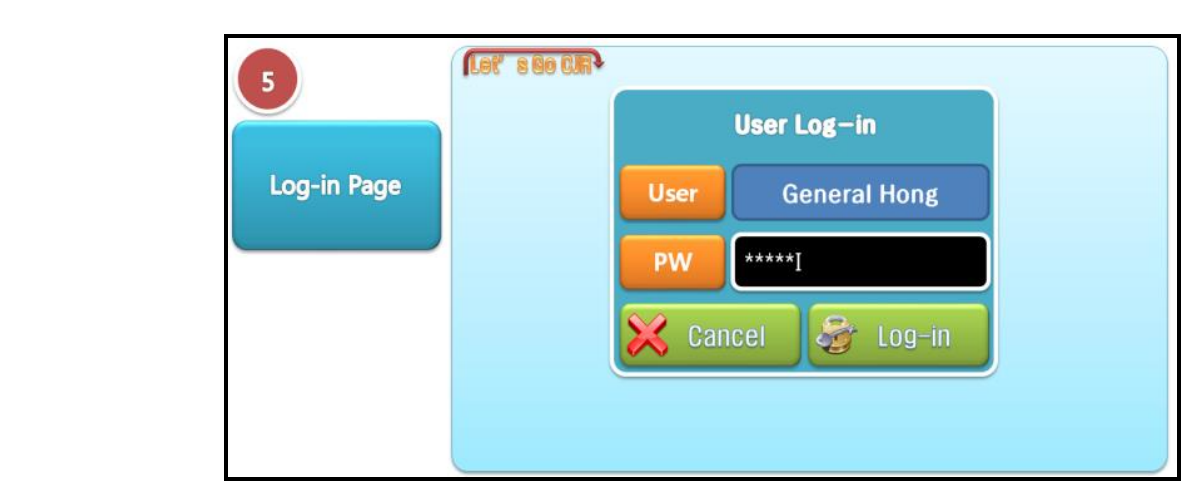

Figure 6. The log-in page of our app

A user will see a main page when he succeed in log-in. Figure 7 shows the main page of our app. The main page has several menus including "Memorial Place", "Leaving Memories", "Recommending Tour Course", "What at Choong-Jang-Ro?", "Modifying User Info". First, 
"Memorial Place" is the menu for remembering places where we often went in the past. Second, "Leaving Memories" is the menu for leaving traces of visitors. So, tourists visit famous places on Choong-Jang-Ro, and can write postscripts on the log of our app. Third, "What at Choong-Jang-Ro?" is the menu for providing information of places related to shopping, performance, famous restaurant. Finally, "Recommending Tour Course" is the menu for visitors who don't know Choong-Jang-Ro well.

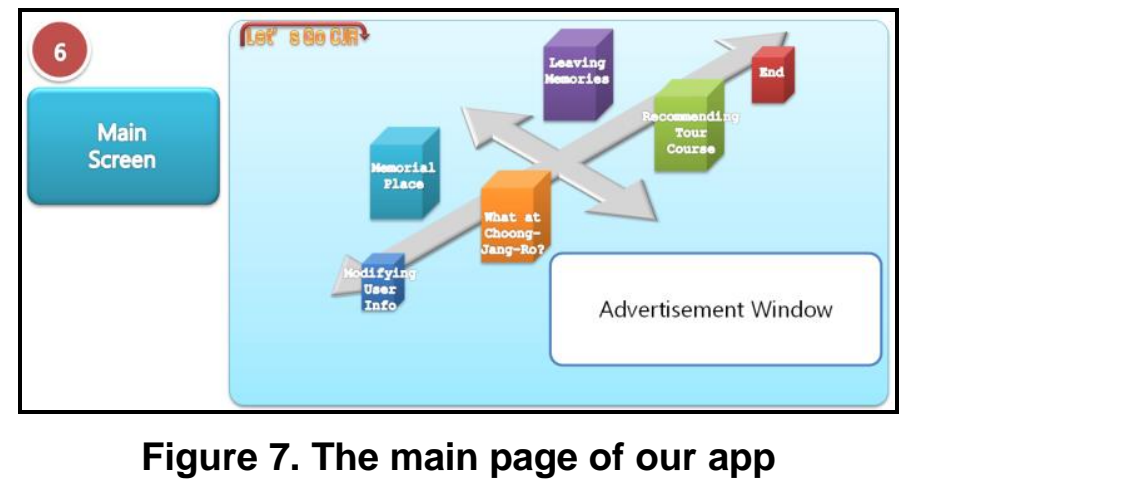

A user will see a part 1 page of memorial place if hesclicks "Memorial Place" menu on main page. Figure 8 shows outline of the part 1 page. Inthe first, our app mark user's position using GPS on the map. The list of picture in the below includes the places where a user can go on the current map, and is scrofled left and right. If a user clicks a certain picture in the list, he will enter a part 2 page.

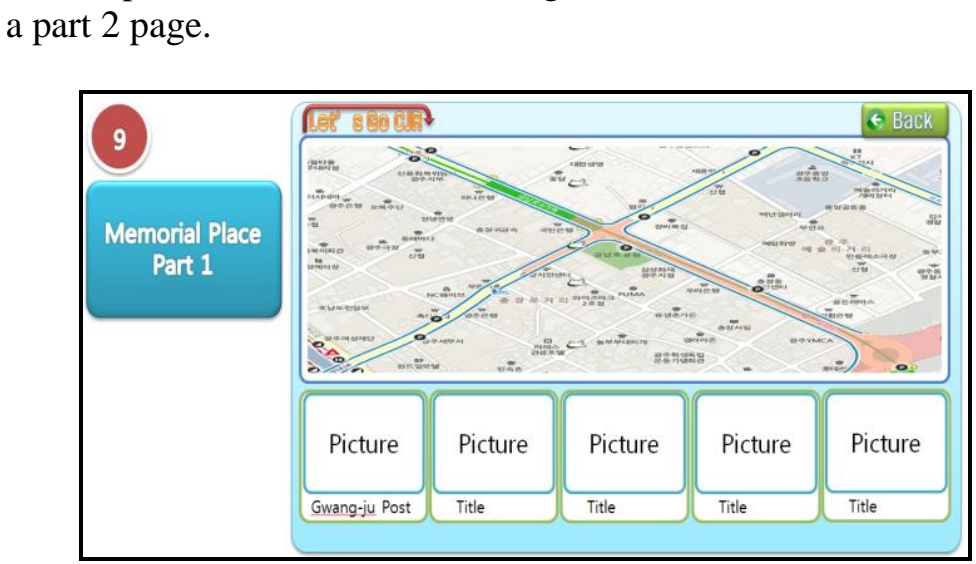

Figure 8. The part 1 page of memorial place

A user will see a part 2 page of memorial place if he clicks a certain picture on the upper page. Figure 9 shows outline of the part 2 page. This page explains the place related to that picture in detail and provides information. Map window displays map near the place. The arrow mark indicates the direction from a user's position to the place. 


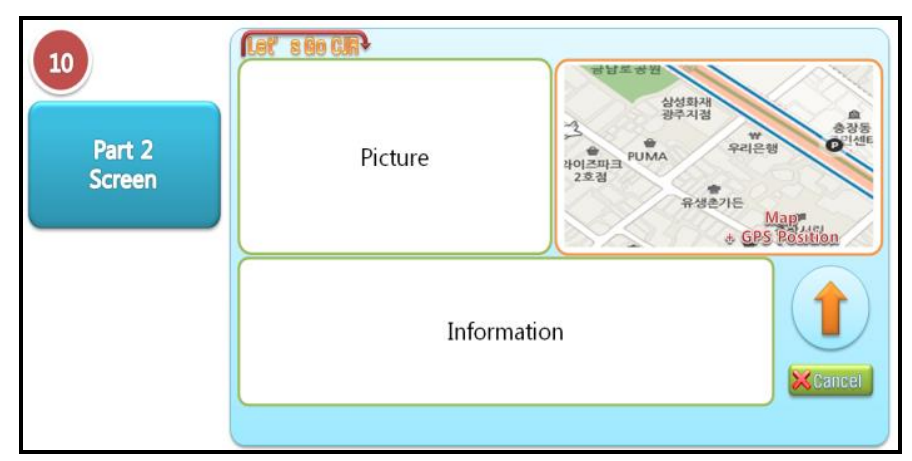

Figure 9. The part 2 page of memorial place

A user will see a part 1 page of "What at Choong-Jang-Ro?" menu if he clicks it on the main page in Figure 7. The outline of the page is shown in Figure 10. This page includes various information which a user can enjoy himself at Choong-Jang Ro such as performance, shopping, food, and accommodation. If a user want to know specific information list, he just need to click the appropriate button in the left. After that, the infornation list related to the button is displayed in the right window. If a user clicks one record in the list, our app move into the part 2 page which he have seen in.Figure 9.

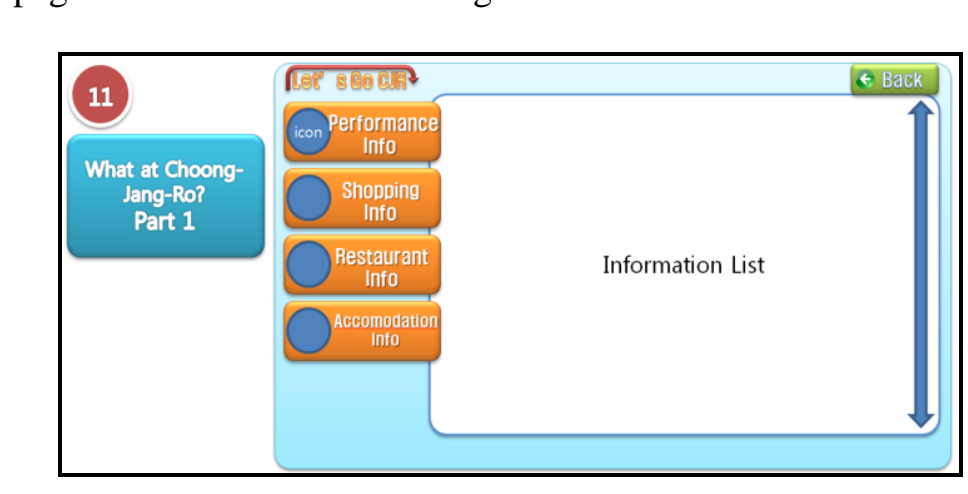

Figure 10. The part 1 page of "What at Choong-Jang-Ro" menu

A user will see hag input page of "Leaving Memories" menu if he clicks it on the main page in Figure 7 and selects a specific location. The outline of the page is shown in Figure 11. A user just neecto take a picture of a specific location and write a log related to the picture. So, if other people visit this place on our app, they can see the tag contents which the upper user made before.

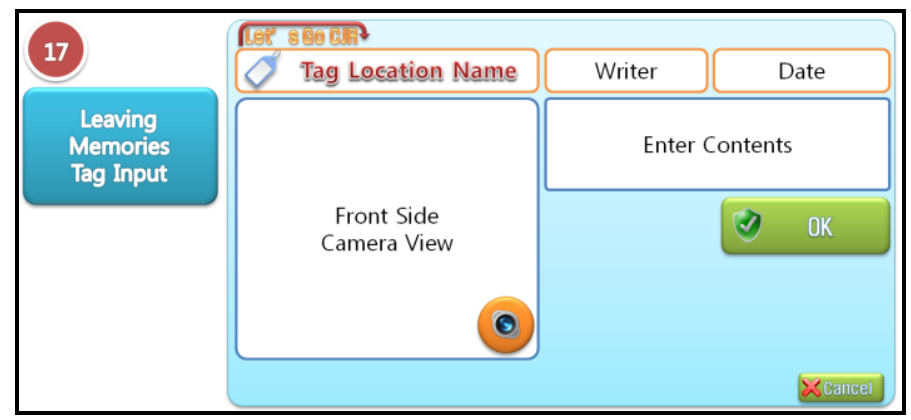

Figure 11. The tag input page of "Leaving Memories" menu 
A user will see a part 1 page of "Recommending Tour Course" menu if he clicks it on the main page in Figure 7. The outline of the page is shown in Figure 12. Each course has its information list. If a user clicks a place on the information list, our app move into a detailed page for the selected place.

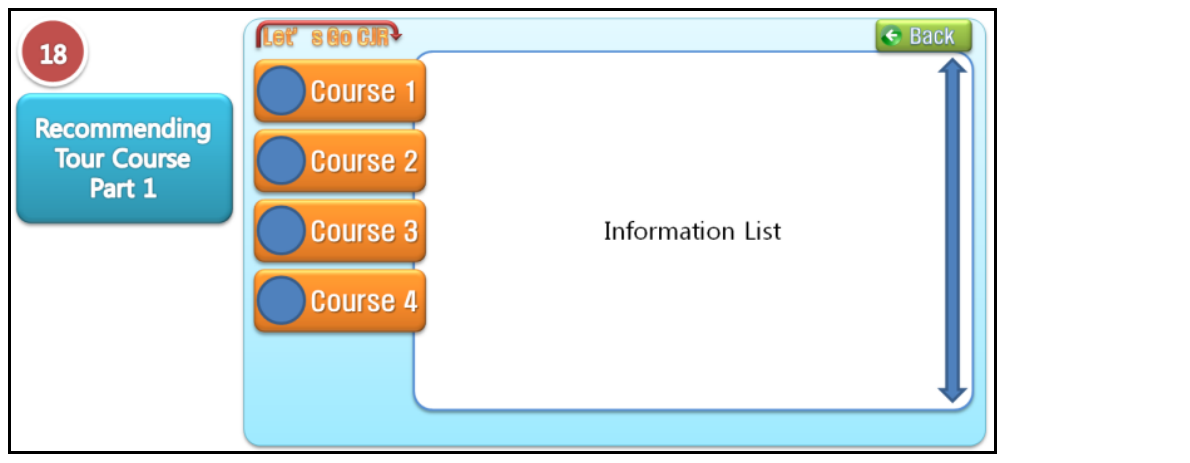

Figure 12. The part 1 page of "Recommending Tour Course" menu

\section{Database design}

The database of our app has six tables. The tables are LC_USER_INFO, LC_DATA_INFO, LC_IMG_INFO, LC_TAG, LC_COMMON_CODE, YO_LC_INFO. We explain each table in detail.

First, LC_USER_INFO is the table on which our app stores several data related to a user. Table 1 shows the attributes of that table.

\section{Table 1. Design of LC_USER_INFO}

\begin{tabular}{|c|c|c|c|}
\hline $\mathrm{NO}$ & Attribute Nane & Type & Description \\
\hline 1 & User id A & Varchar(20) & User's identifier \\
\hline 2 & User_nm & Varchar(20) & User's name \\
\hline 3 & User nichm & Varchar(20) & User's nickname \\
\hline 4 & User_type & Varchar(03) & User's type \\
\hline 5 & User_pw & Varchar(255) & User's password \\
\hline 6 & Jm_bh & Varchar(255) & Social security number \\
\hline 7 & User_pno & Varchar(255) & User's phone number \\
\hline 8 & Device_id & Varchar(255) & Unique identifier of user's phone \\
\hline 9 & User wype & $\operatorname{Varchar}(03)$ & Telecommunication company \\
\hline 10 & Cr_date & Date & Registration date of a user \\
\hline 11 & End_edit_date & Date & The most recent update date \\
\hline & End_edit_id & Varchar(20) & The most recent update person \\
\hline
\end{tabular}

Second, LC_DATA_INFO is the table on which our app stores text information related to Choong-Jang-Ro. Table 2 shows the attributes of that table.

Table 2. Design of LC_DATA_INFO

\begin{tabular}{|c|l|c|l|}
\hline NO & \multicolumn{1}{|c|}{ Attribute Name } & Type & \multicolumn{1}{|c|}{ Description } \\
\hline 1 & Lc_dtype & Varchar(03) & Type of text information \\
\hline 2 & Lc_dcode & Varchar(10) & Code of text information \\
\hline 3 & D_title & Varchar(255) & Title of text information \\
\hline 4 & D_text & Text(5000) & Main text information \\
\hline 5 & Gps_lat & Float(10,6) & Latitude position of text information \\
\hline 6 & Gps_lon & Float $(10,6)$ & Longitude position of text information \\
\hline
\end{tabular}


Third, LC_IMG_INFO is the table on which our app stores image information related to Choong-Jang-Ro. Table 3 shows the attributes of that table.

Table 3. Design of LC_IMG_INFO

\begin{tabular}{|c|l|c|l|}
\hline NO & \multicolumn{1}{|c|}{ Attribute Name } & Type & \multicolumn{1}{c|}{ Description } \\
\hline 1 & Lc_dtype & Varchar(03) & Type of image information \\
\hline 2 & Lc_dcode & Varchar(10) & Code of image information \\
\hline 3 & Sno & $\operatorname{Int}(3)$ & Sequence number of image information \\
\hline 4 & Img_folder & Varchar(255) & Folder of an image file \\
\hline 5 & Img_filename & Varchar(255) & Name of an image file \\
\hline 6 & Img_comment & Varchar(255) & Comment about an image \\
\hline
\end{tabular}

Fourth, LC_TAG is the table on which our app stores tag information for a user to write. Table 4 shows the attributes of that table.

Table 4. Design of LCTAG

\begin{tabular}{|c|l|c|c|}
\hline NO & Attribute Name & Type & \\
\hline 1 & Lc_dcode & Varchar(10) & Code of tag \\
\hline 2 & Tag_sn & Int(8) & Tag sequence number \\
\hline 3 & Lc_dtype & Varchar(03) & Tag type \\
\hline 4 & D_title & Varchar(100) & Tag title \\
\hline 5 & D_text & Varchar(255) & Main text of tag \\
\hline 6 & Cr_id & Varchar(20) & W.iter's id \\
\hline 7 & Cr_nicnm & Varchar(20) & Writer's nickname \\
\hline 8 & State_icon & Varchar(03) & Tag state \\
\hline 9 & Cr_date & Date__ & Date to write tag at \\
\hline
\end{tabular}

Fifth, UC_LC_INFO is the table on which our app stores simple user information. Unlike the other tables, this table exists at user's phone. Table 5 shows the attributes of that table.

Table 5. Design of UC_LC_INFO

\begin{tabular}{|c|l|l|l|}
\hline NO & \multicolumn{1}{|c|}{ Attribute Name } & Type & \multicolumn{1}{c|}{ Description } \\
\hline 1 & User_id & Varchar(20) & User's identifier \\
\hline 2 & User_nicnm & Varchar(20) & User's nickname \\
\hline
\end{tabular}

Sixth, LCACOMMON_CODE is the table on which our app stores the data about common code. Table 6 shows the attributes of that table.

Table 6. Design of LC_COMMON_CODE

\begin{tabular}{|c|l|c|l|}
\hline NQ & \multicolumn{1}{|c|}{ Attribute Name } & Type & \multicolumn{1}{c|}{ Description } \\
\hline 1 & Lc_type & Varchar(10) & Type of common code \\
\hline 2 & Lc_code & Varchar(03) & Identifier of common code \\
\hline 3 & Lc_name & Varchar(30) & Name of common code \\
\hline 4 & Lc_svalue & Varchar(256) & Character value of common code \\
\hline 5 & Lc_nvalue & Number(10) & Numeric value of common code \\
\hline 6 & Lc_note & Varchar(256) & Comment for common code \\
\hline
\end{tabular}




\section{Implementation}

We implement our app on PhoneGap which is a leading hybrid platform. PhoneGap supports various smart phone platform including iOS, Android. So, our app is executed on any smart phone.

Figure 13 shows an example page for memorial places of Choong-Jang-Ro based on augmented reality. In the first, memorial places of Choong-Jang-Ro is marked on the map. Then, if a user points out one of marker places, related explanation and picture appears.

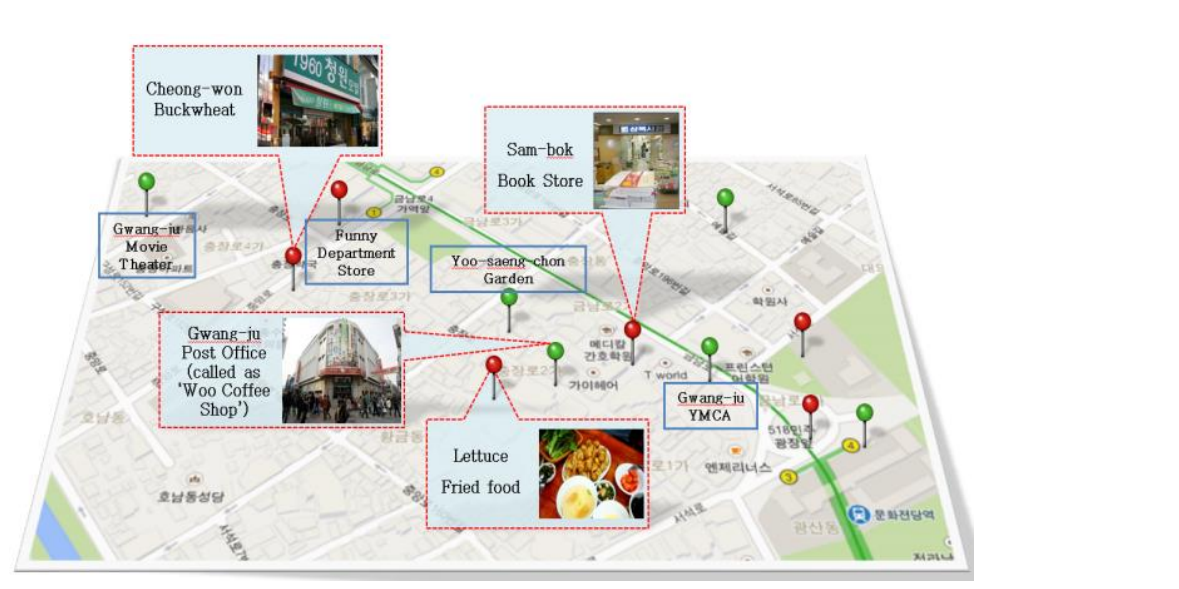

Figure 13. An example page for memorial places of Choong-Jang-Ro

\section{Conclusion and Futuree work}

Choong-Jang-Ro has been a central place for a long time in Gwang-ju, but it was on the decline recently_For activation of Choong-Jang-Ro, an app to let people know it on state-ofthe-art devices needed to be developed. To achieve this objective, we proposed the prototype design of an app for Choong-Jang-Ro. Our proposed app had 4 services including a story telling service, a service to provide information related to Choong-Jang-Ro, a social network service, and a service to recommend tour course. Our app utilized a technology of augmented reality. We implemented our app based on the design of user interface and database. In the future, we will upgrade our app to launch on a commercial market.

\section{Acknowledgements}

This /research was supported by Smart Contents Creation Project through the Gwangju Information \& Culture Industry Promotion Agency (GITCT) funded by the Ministry of Science, ICT and Future Planning (2013-A-007).

\section{References}

[1] http://utour.gwangju.go.kr/utour/culture.do?S=S01\&M=060600000000\&b_code=CULTURE_KOR \&tour_cg=TOUR022\&act=view\&list_no=322.

[2] http://en.wikipedia.org/wiki/Augmented_reality . 


\section{Authors}

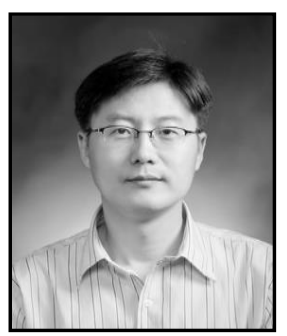

\section{Seong-Ho Lee}

He received B.S., M.S., Ph.D. degrees from Chonnam National University, Gwang-ju, Korea, in 1995, 1999, and 2005, respectively, all in computer science. In 2011-2013, he was with the Information Industry Research Institute at the Mokpo National University, Mu-an, Korea, as a researcher. From July 2013, he has joined as a research head at Uniyersal Community Co. Ltd., Gwang-ju, Korea. His research interests include big data processing, cloud computing, NFC technology, and security.

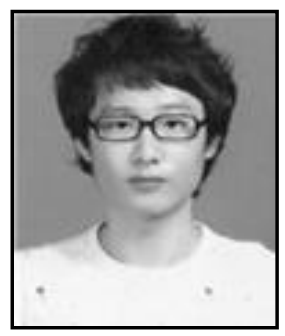

\section{JongMin Moon}

He received B.S. degree from Chosun University, Gwang-ju, Korea, in 2012, in computer engineering. From November 2012, he has joined as a researcher at Universal Community C6. Ltd., Gwang-ju, Korea. Since joining at the UC, he exeeuted several projects related to secured voice communication using SIP and Hadoop-based big data processing. His research interestrmolude cloud computing and network security.

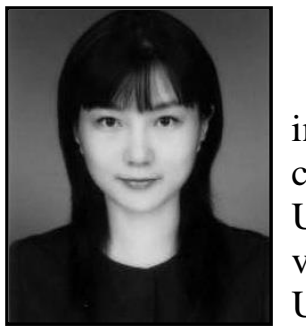

\section{Hyeo-Won No}

She received M.S. degree from Honam University, Gwang-ju, Korea, in 2002, in multimedia engineering. From 2005, she has entered in Ph.D. course of the Department of Image Processing at Chonbuk National University, Jeon-ju, Korea. From March 2011, she has served as a visiting professor at the Department of Internet Contents, Honam Oniversity, Gwang-ju, Korea. Her research interests include image

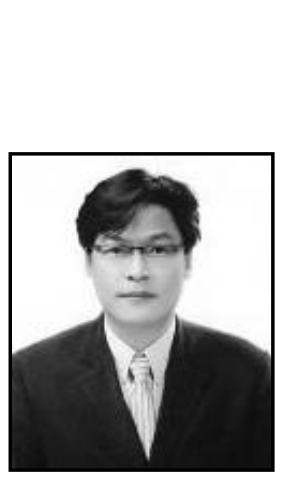
processing, Web 3D, web service, UX\&UI.

\section{Su-Yong Lee}

He was born in Gwang-Ju, Republic of KOREA, on Feb. 29. 1972. He received the BArch, M.A, and Ph.D. degree in Department of Architectural Engineering from Chonnam National University, KOREA in 1998, 2003, and 2010 respectively. He has worked professor in dept. of Architectural Engineering. Dong-Shin Univ. Sep. 2005 to Aug. 2009. He interests in Environment-Friendly Design, Urban Regeneration. He is a member of AIK, KHA, KIRA. 


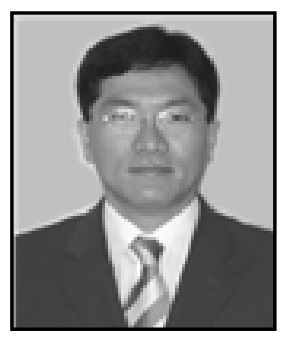

\section{HyeongGyun Kim}

He is an adjunct professor at the Department of Computer Engineering, Chosun University, Gwang-ju, Korea. He received Ph.D. degree in computer engineering from Chosun University in 2004 and M.S. degree in computer science from Chosun University in 1998. In 2004, he was with the Department of Computer Information at the Dong-kang College, Gwang-ju, Korea, as a visiting professor. In 2007, he was with the Engineering Innovation Center at the Honam University, Gwang-ju, Korea. His research interests include image processing, smart phone application, cloud computing, big data, and NFC.

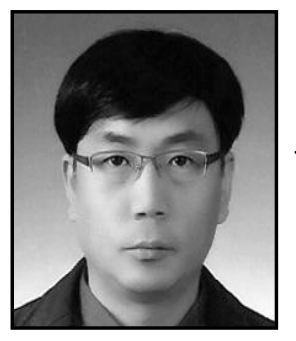

\section{Ki-Bong Joung}

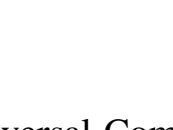

He is a development head at Universal Community Co. Ltd., Gwangju, Korea. He received Ph.D degree in computer engineering from Chosun University in 2003 and M.S. degree $1 n$ industrial engineering from Chosun University in 1997. From January 2007 to June 2013, he has worked as a leader of school affairs at the Center of Information Management, Mokpo Seience Unversity, Mokpo, Korea. His research interests include cloud computing, big data, and NFC. 
International Journal of Multimedia and Ubiquitous Engineering Vol.9, No.2 (2014)

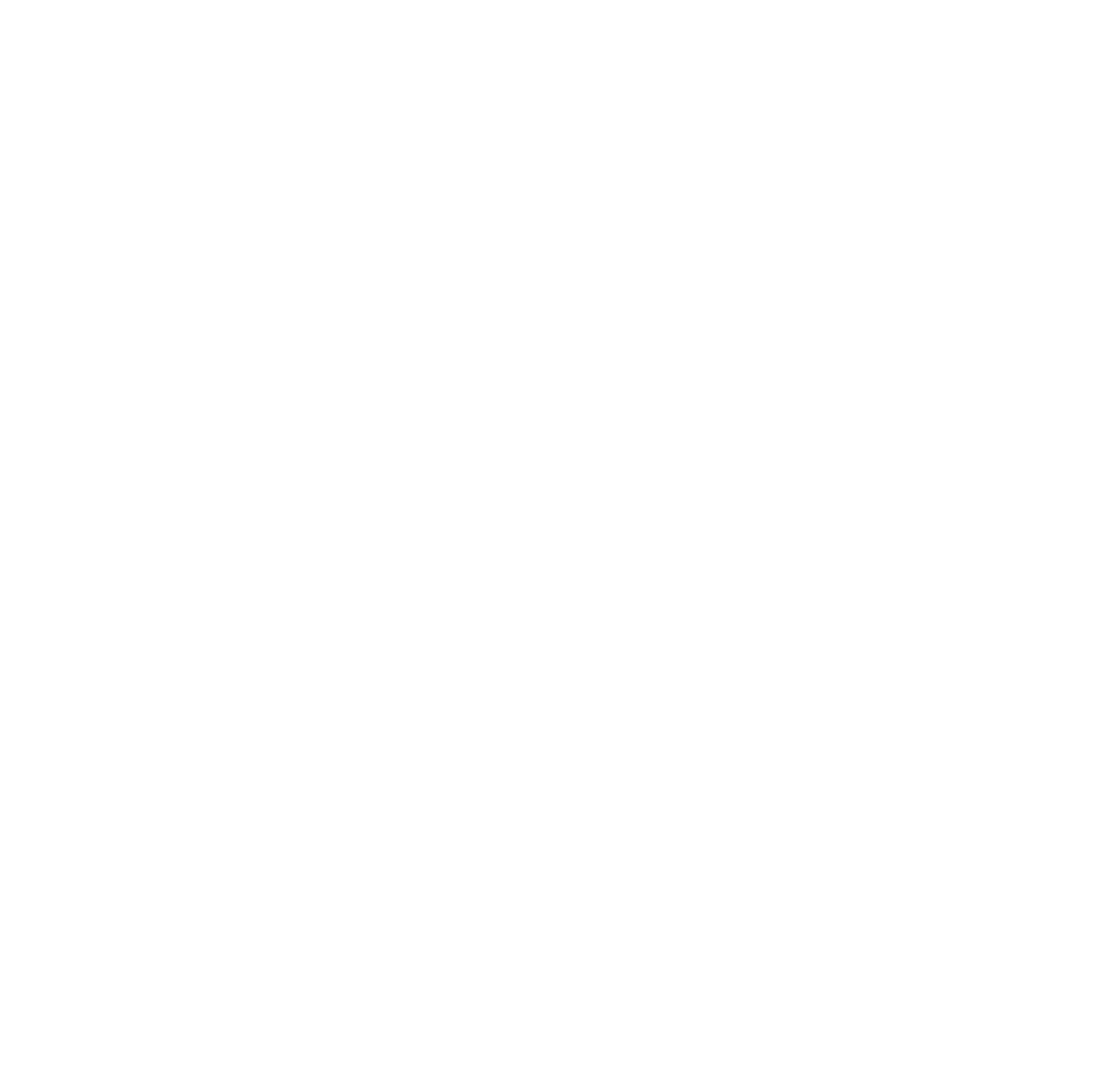

